Revue des patrimoines

$33 \mid 2017$

Patrimoine culturel immatériel et institutions patrimoniales

\title{
Les patrimoines de l'agropastoralisme dans les Hautes-Alpes
}

The heritage of agropastoralism in the Hautes-Alpes department

\section{Philippe Grandvoinnet}

\section{(2) OpenEdition}

\section{Journals}

Édition électronique

URL : http://journals.openedition.org/insitu/15545

DOI : $10.4000 /$ insitu. 75545

ISSN : 1630-7305

Éditeur

Ministère de la culture

Référence électronique

Philippe Grandvoinnet, « Les patrimoines de l'agropastoralisme dans les Hautes-Alpes », In Situ [En

ligne], 33 | 2017, mis en ligne le 03 novembre 2017, consulté le 19 avril 2019. URL : http://

journals.openedition.org/insitu/15545; DOI : 10.4000/insitu.15545

Ce document a été généré automatiquement le 19 avril 2019

\section{cc) (†)}

In Situ Revues des patrimoines est mis à disposition selon les termes de la licence Creative Commons Attribution - Pas d'Utilisation Commerciale - Pas de Modification 4.0 International. 


\title{
Les patrimoines de l'agropastoralisme dans les Hautes- Alpes
}

The heritage of agropastoralism in the Hautes-Alpes department

\author{
Philippe Grandvoinnet
}

1 Parfois qualifiées de conservatoire des traditions agropastorales, les Hautes-Alpes sont un département rural marqué par un climat contrasté et un relief abrupt. Ces conditions géographiques et climatiques ont conduit les populations à développer des stratégies agricoles collectives, ancrées dans une forte saisonnalité. Cela s'est traduit concrètement dans les usages du territoire (étagement des alpages sur la montagne, multiplication des hameaux d'estive, mise en commun des espaces de pâture) et dans la culture populaire (oralité, artisanat, colportage, etc.) ${ }^{1}$.

\section{Habiter les étages de la montagne}

2 L'usage des pâturages d'altitude est attesté dans les Alpes depuis le Néolithique ${ }^{2}$ : pour optimiser la ressource en herbe - dont la croissance s'étale tout au long de la saison estivale selon l'altitude - les troupeaux changent de pâturage et montent progressivement jusqu'aux pelouses alpines les plus hautes. Dans des vallées où les surfaces agricoles exploitables sont réduites et les rendements plus faibles qu'ailleurs en raison d'une saison végétative courte, l'élevage assurait traditionnellement un complément indispensable à la survie des populations. Qu'il s'agisse de transhumance sur de longues distances (entre la Provence et les Alpes par exemple) ou de déplacements de troupeaux sur le territoire de la communauté, les conditions géographiques et climatiques déterminent les parcours. Au début de l'été, les troupeaux qui ont hiverné dans la plaine ou les vallées montent dans les alpages, d'abord dans les «quartiers» de moyenne altitude (fig. 1), où l'herbe est précoce, puis plus haut, jusque dans les derniers quartiers où le troupeau finit la saison avant de redescendre. Au milieu du XIII ${ }^{\mathrm{e}}$ siècle, les 
montagnes du Queyras accueillaient durant l'été jusqu'à 12000 moutons, dont le tiers environ venait d'autres territoires ${ }^{3}$. En saison d'estive, la communauté vivait au rythme que lui imposaient l'agriculture et l'élevage, ce qui impliquait de nombreux déplacements entre les villages (permanents ou saisonniers) et les espaces agricoles et pastoraux dispersés dans le finage communal.

Figure 1

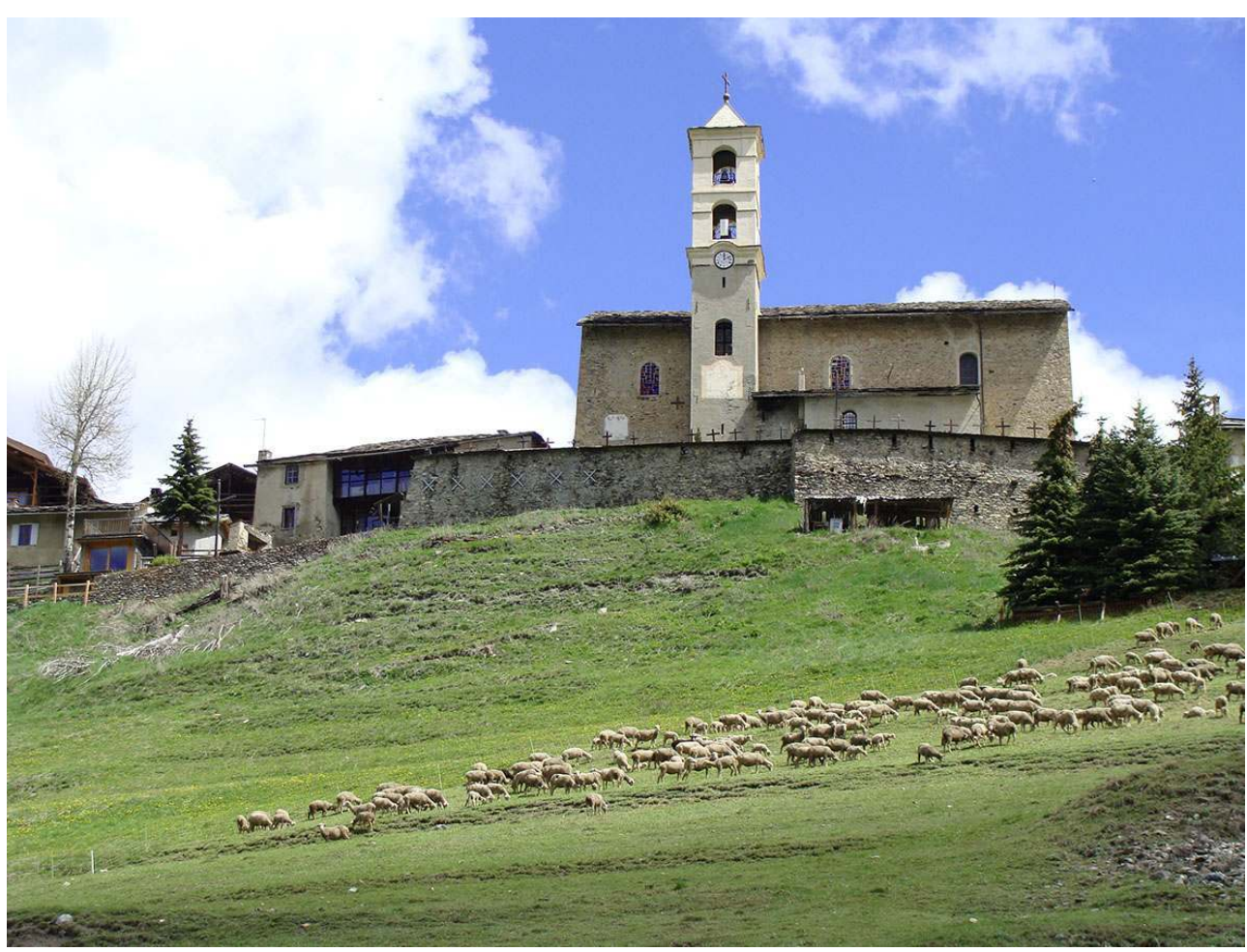

Saint-Véran (2040 m) : troupeau de moutons au pied du village à la fin du printemps.

Phot. Philippe Grandvoinnet, 2014. (C) UDAP 05.

3 Le haut Dauphiné, et plus particulièrement le nord des Hautes-Alpes (Briançonnais, Queyras, Embrunais), a donc une longue tradition pastorale dont témoignent les paysages, le patrimoine bâti et les traditions populaires. Les habitants des hautes vallées alpines ont développé au fil des siècles des savoirs propres qui leur ont permis d'exploiter au mieux les ressources disponibles selon les terroirs et les époques de l'année ${ }^{4}$. Les communautés changeaient d'habitat au cours de l'année et évoluaient entre le village permanent et plusieurs groupements d'habitat saisonnier, hameaux d'estive ou chalets isolés dans les alpages. L'habitat permanent est implanté en fond de vallée ou en pied de versant, à l'adret pour bénéficier d'une meilleure exposition, dans des sites protégés des risques naturels (crues, avalanches, chutes de blocs). Pour économiser l'espace cultivable, les villages se sont établis sur des pentes abruptes, des escarpements rocheux ou en bordure d'éboulis torrentiels, où la valeur agronomique des sols est faible. Cet habitat permanent (qui peut n'être utilisé que pendant les mois d'hiver mais constitue le cœur de la communauté) est situé au centre d'un terroir agricole composé de potagers, de champs cultivés, de prés de fauche et de forêts. L'habitat saisonnier est réparti sur les différents étages de la montagne. Son organisation dépend à la fois des conditions naturelles (relief, exposition, altitude) et du système d'exploitation adopté par la communauté5. 
$4 \mathrm{Au}$ Moyen Âge et jusqu'à l'époque contemporaine, le terme "montagne » désigne les alpages ou la maison d'estive ${ }^{6}$. Il existe traditionnellement deux modes d'exploitation des alpages, étudiés dès le début $\mathrm{du} \mathrm{Xx}^{\mathrm{e}}$ siècle par les géographes et les ethnologues qui adoptent les termes savoyards de "grande » et de "petite montagne " pour les décrire Dans la grande montagne, les membres de la communauté regroupent leurs bêtes en un troupeau communal confié à des alpagistes professionnels ou «montagniers ${ }^{8}$ " qui en assurent la surveillance et les soins la saison durant. Le bétail - ovins ou bovins, en proportion variable selon les secteurs et les époques - estive sur les alpages communaux et le lait est transformé dans de grands chalets collectifs. Les paysans peuvent ainsi se consacrer pleinement aux travaux agricoles, moisson et fenaison, qui les occupent plus bas dans la vallée. Dans la petite montagne, chaque famille estive avec son bétail dans un chalet pour toute la saison, le bétail est gardé à tour de rôle par un membre de la communauté ; ceux qui restent au village assurent les travaux agricoles tandis que le foin est engrangé dans les chalets d'où il est descendu plus tard par traîneau.

5 Ce mode d'exploitation a été pratiqué jusque dans les années 1960 et reste présent dans la mémoire collective des communautés, comme en témoigne, en 2005 encore, une habitante de Cervières (Hautes-Alpes), Élisabeth Faure-Vincent :

On languissait de se changer* [voir glossaire en note ${ }^{9}$ ]. Tout se passait à pied, avec des charrettes pour le foin, des tombereaux, des mulets. Dans les tombereaux, on mettait les agneaux, les poules et les couvées, le chat, dans des cages. Le linge personnel, des provisions. On poussait les moutons, les brebis, les vaches. Les grosses machines restaient à la montagne* : les charrues, les batteuses, les fléaux, les chaudrons aussi, la vaisselle, le linge de maison, etc.

On montait au mois de mai dans la vallée des Fonts (fig. 2), pour la première montagne, la plus haute. Nous, notre montagne était au Rif Tord. À l'automne, aux premières neiges, on descendait dans la montagne basse : le Laus ou Terre Rouge. Tout le monde était propriétaire de sa maison, au village et en haut. Et chacun avait un jardin, en haut aussi. Fin mai, on plantait du seigle, des pommes de terre. En automne, du blé tramois*. On le coupait quand il jaunissait, vers juillet-août, et on le faisait mûrir en gerbes sur les plans*, dans le grenier. Dans les maisons, les vaches étaient d'un côté. On dormait à l'écurie, dans des lits surélevés, au-dessus d'un espace pour les réserves ou les brebis. Il y avait une cuisine propre, séparée de l'écurie, pour travailler le lait. Et une cave, pour conserver au frais les fromages, le beurre, le lait, les œufs. Les vaches, on les rentrait tout l'été, pour la traite du soir, la nuit et la traite du matin. On les gardait à tour de rôle. Il y avait une vacherie* dans chaque village*.

Les moutons restaient dehors, gardés par un berger. L'été, toutes les bêtes étaient à la montagne. Quand j'étais ménagère*, je remontais tous les soirs pour m'en occuper et je redescendais le matin pour travailler en bas. En août, on restait làhaut pour les foins. Il n'y avait plus personne à Cervières. On fauchait à la faux et au râteau. Puis on descendait les trousses* au village. Dans la grande grange, on laissait un peu de foin, si on en avait beaucoup. Il y avait un plan* où on mettait les fourrages, un plan où on mettait les récoltes, mais on descendait surtout en bas pour l'hiver. On était vraiment contents de partir à la montagne. Car il y avait plus d'espace en haut, des prés... on se voyait plus, entre voisins. On allait ramasser des fleurs, des framboises, des fraises, des airelles, des champignons, des violettes pour faire de la tisane. Une fois par mois, chaque pays avait sa messe... Au Bourget, c'était la Transfiguration. À la Chau, Sainte-Élisabeth...Monsieur le Curé montait dire la messe, et on faisait une petite surprise, des croquants... Chacun son tour. La montagne, c'était la liberté... 


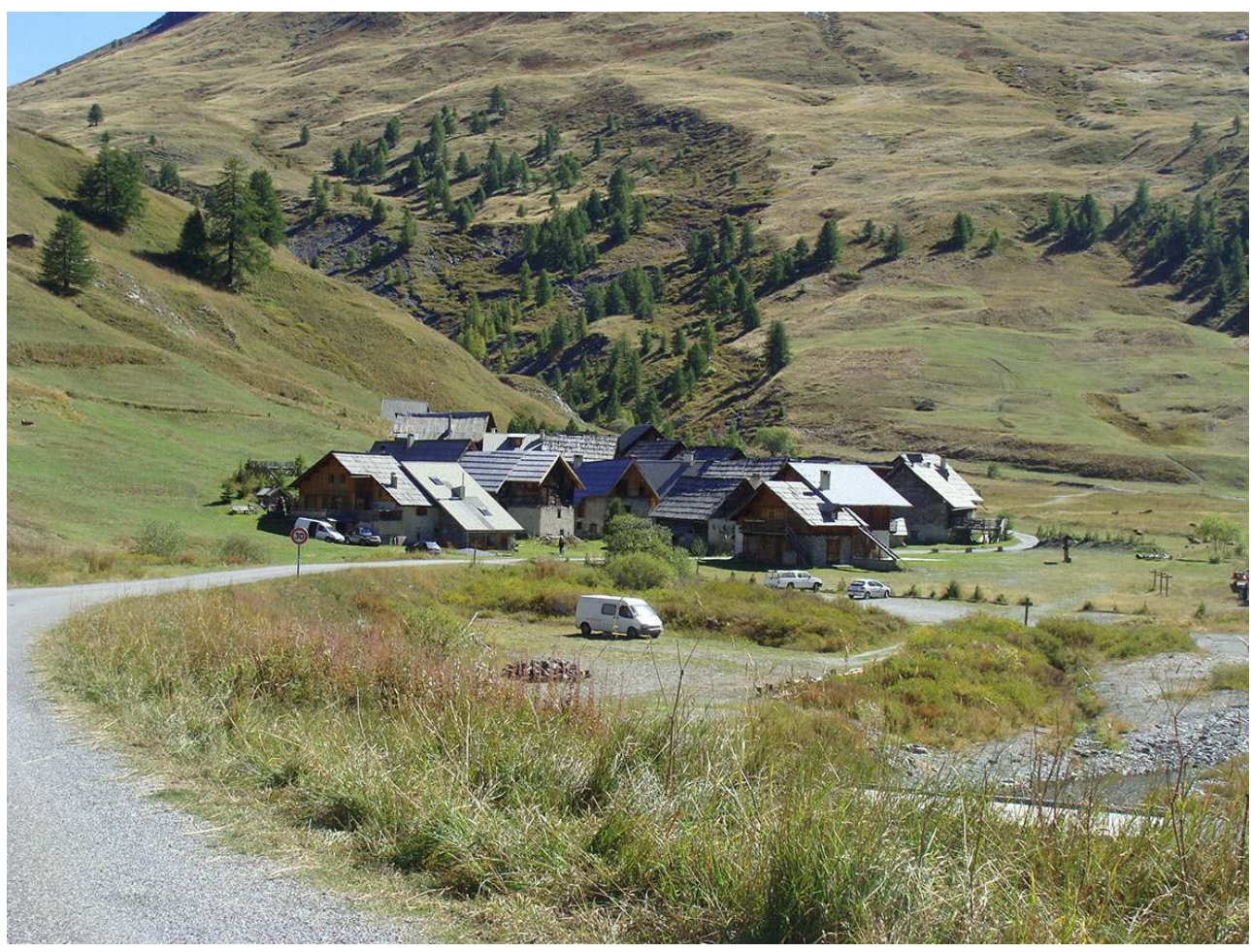

CERVIÈres, hAMEAU des FONTS (2 $000 \mathrm{M})$.

Phot. Philippe Grandvoinnet, 2014. (C) UDAP 05.

\section{Jardiner la montagne}

6 L'utilisation rationnelle et intensive de l'espace utile en montagne repose sur des aménagements ingénieux qui améliorent la productivité des terres et facilitent leur exploitation: épierrage des champs et constitution de pierriers ou «clapiers", construction de banquettes et de terrasses pour retenir la terre et faciliter la culture céréalière, création de réseaux pour l'irrigation des champs cultivés et des prés de fauche, etc. Afin d'optimiser les ressources disponibles sur un territoire contraint et limité et répartir équitablement la ressource entre les membres de la communauté, chaque famille dispose de parcelles dispersées sur la totalité du finage communal. Cette fragmentation du parcellaire - accentuée par les divisions résultant des successions - s'accompagne d'une multiplication des bâtiments agricoles aux différents étages de la montagne, occupés successivement au cours de la saison ${ }^{10}$.

7 Ces territoires agricoles de haute-montagne font l'objet d'une attention méticuleuse qui confine au jardinage (fig. 3) : les chemins sont stabilisés par des murets de soutènement, les prés de fauche sont irrigués par des canaux, l'herbe - denrée précieuse - est fauchée partout où elle n'est pas laissée en pâture au bétail, champs et prés sont protégés de la divagation des troupeaux au moyen de murets ou de clôtures ; plus haut, la pelouse alpine est entretenue par le pâturage des troupeaux, les bêtes laissant émerger ça et là, en bouquets, les plantes toxiques ou ligneuses qu'elles dédaignent. Certaines plantes - tel le vératre - sont parfois arrachées à la main par les bergers pour éviter que les bêtes ne s'intoxiquent $^{11}$. Sur les parcelles agricoles les plus raides, la terre est remontée chaque 
année à dos d'homme ou de mulet. Ces terroirs ont été organisés puis entretenus par des générations de paysans selon des pratiques agropastorales qui ont pu varier d'une vallée à l'autre selon les époques, la qualité agronomique du finage, le statut du foncier et les traditions locales. Si leur valeur est avant tout agricole (depuis 1985, la loi Montagne pose comme principe intangible la protection des surfaces agricoles), leur qualité paysagère est indéniable et reconnue depuis de nombreuses années.

Figure 3

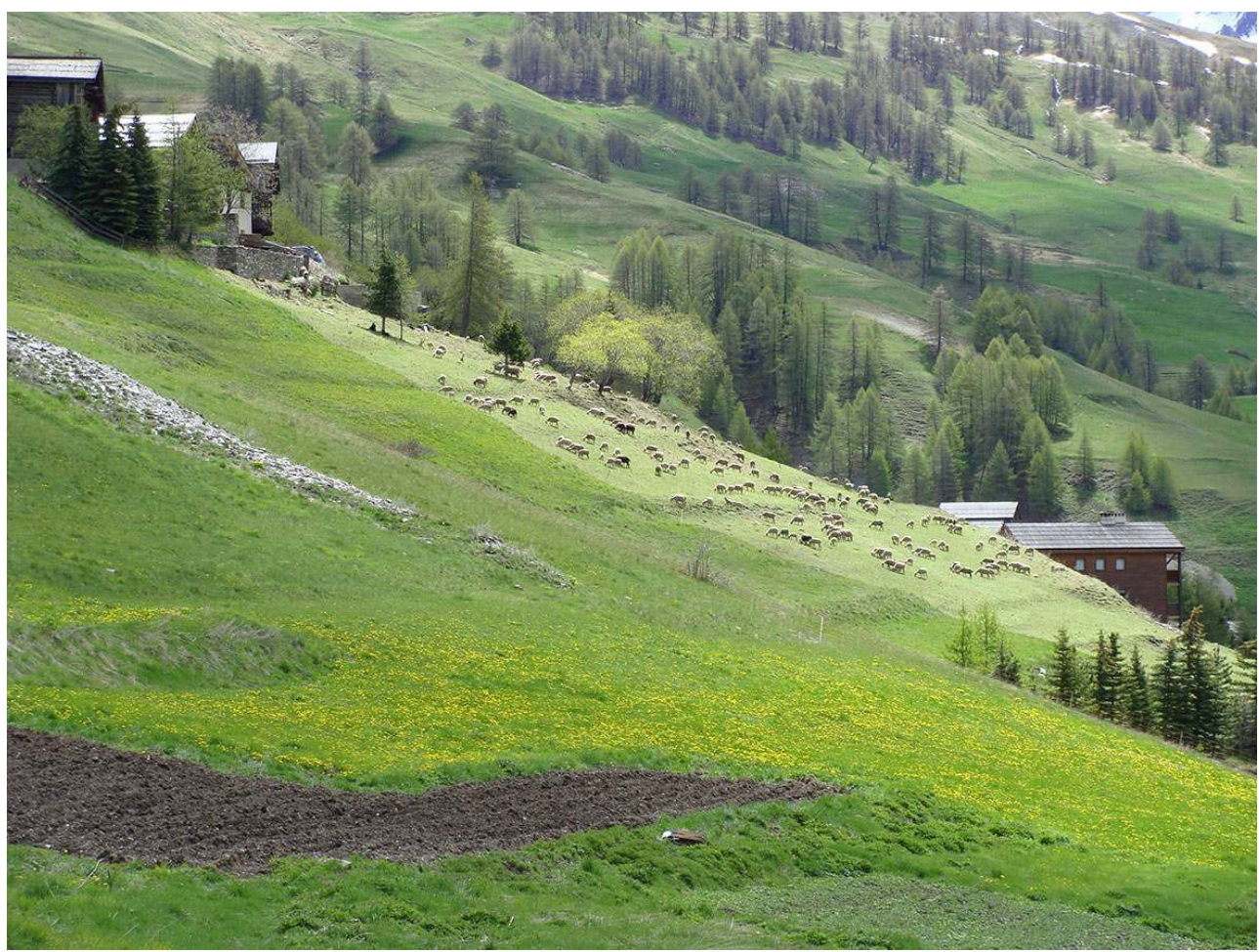

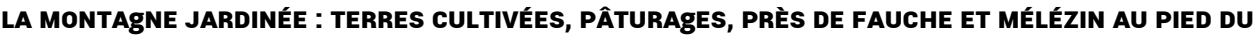
VILLAgE DE SAINT-VÉRAN.

Phot. Philippe Grandvoinnet, 2014. C) UDAP 05.

8 Ces techniques agropastorales ont interagi fortement avec la forêt : à l'essartage intensif du Moyen Âge (extension des zones cultivées mais aussi des prairies d'altitude) a succédé une stricte protection des secteurs boisés proches des villages, mis en défens pour assurer l'autonomie des communautés en bois d'œuvre et bois de chauffe. Dans toutes les Alpes du Sud, la cembraie ${ }^{12}$, qui dominait les étages subalpin et alpin, a ainsi régressé au profit du mélézin, privilégié par les populations car il permet d'associer sur un même espace exploitation forestière et pâture, selon le principe de l'agroforesterie. Essence de lumière, emblématique des Alpes du Sud, le mélèze est un résineux à aiguilles caduques qui a la particularité d'offrir un sous-bois lumineux où l'herbe croît suffisamment pour constituer des pâturages d'appoint. Le mélèze donne par ailleurs un bois d'œuvre de qualité supérieure, imputrescible, utilisable en charpente comme en menuiserie et en tabletterie, autant de domaines qui ont fait la réputation des artisans du Queyras notamment. Les fûts de mélèze sont assemblés "pièce sur pièce " pour former les "fustes", qui constituent l'habitat traditionnel de certaines vallées du Queyras et les bâtiments saisonniers de nombreux alpages (fig. 4). D'un point de vue paysager, le mélèze accentue les variations saisonnières : d'une couleur vert tendre au printemps, plus soutenu en été, 
il se pare de teintes de jaune orangé flamboyantes à l'automne et ne laisse voir que son écorce grisâtre en hiver, quand les aiguilles sont tombées. Le couvert forestier est alors diaphane et laisse transparaître le manteau neigeux du sous-bois. Ces boisements, caractéristiques des Alpes du Sud, traduisent le fort ancrage des traditions agropastorales dans le paysage.

Figure 4

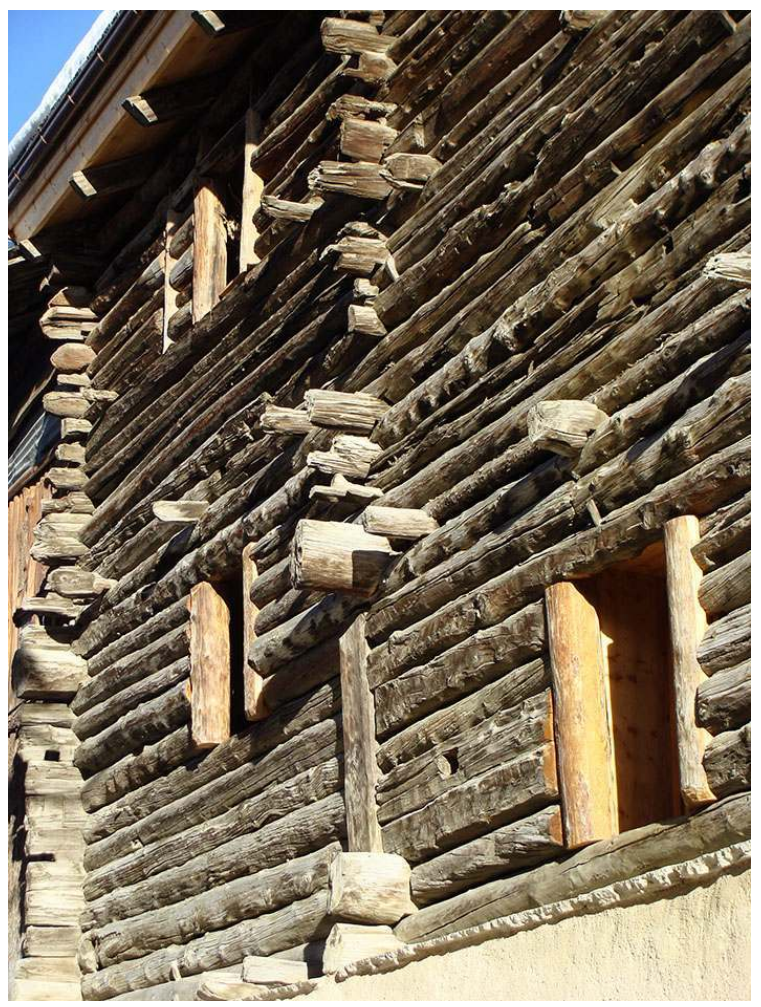

SAINT-VÉRAN, ASSEMBLAgeS TRAdITIONNELS « PIÈCE SUR PIÈCE » D'UNE FUSTE DU VILLAgE.

Phot. Philippe Grandvoinnet, 2014. (C) UDAP 05.

\section{Patrimoines de l'agropastoralisme}

La reconnaissance de la valeur culturelle et paysagère de ces territoires de haute montagne est ancienne. La loi de 1930 sur les sites $^{13}$ a permis de protéger dans les années 1940 un grand nombre de hameaux d'altitude en raison de leur caractère « pittoresque ». Dans les Hautes-Alpes, les principaux groupements de chalets d'alpage de Névache et les hameaux les plus emblématiques de La Grave, sous le col du Lautaret, sont inscrits depuis 1943 (fig. 5). Le Valgaudemar, vallée glaciaire située au sud du massif Écrins-Pelvoux, a fait l'objet d'une inscription complète (soit près de 10000 hectares) en octobre 1946, avec plusieurs de ses hameaux dont certains étaient déjà à l'abandon. Le plus haut village des Alpes, Saint-Véran (2 042 mètres d'altitude), « unique au monde, typique du style du Queyras et pourtant seul de son espèce dans la vallée ${ }^{14}$ » a également été inscrit en octobre 1948. Après un exode rural continu depuis la fin du XIX ${ }^{\mathrm{e}}$ siècle, ces vallées ont connu une baisse démographique marquée, les plus isolées étant bien sûr les plus touchées. La montagne se vide de ses habitants, l'agropastoralisme est en déclin, la déprise des terres agricoles est partout visible et la forêt gagne du terrain, notamment 
sur les versants acquis et plantés par l'État à partir des années 1880 pour ralentir l'érosion ${ }^{15}$. Ces protections ont donc été prises à un moment où les mutations du territoire à l'œuvre depuis plus d'un demi-siècle devenaient plus visibles et où l'on prenait conscience de la fragilité des paysages façonnés par l'agropastoralisme. Il ne s'agissait pas encore d'assurer la continuité des traditions qui ont construit ces paysages mais tout du moins de préserver l'image pittoresque du territoire qu'elles ont produit.

Figure 5

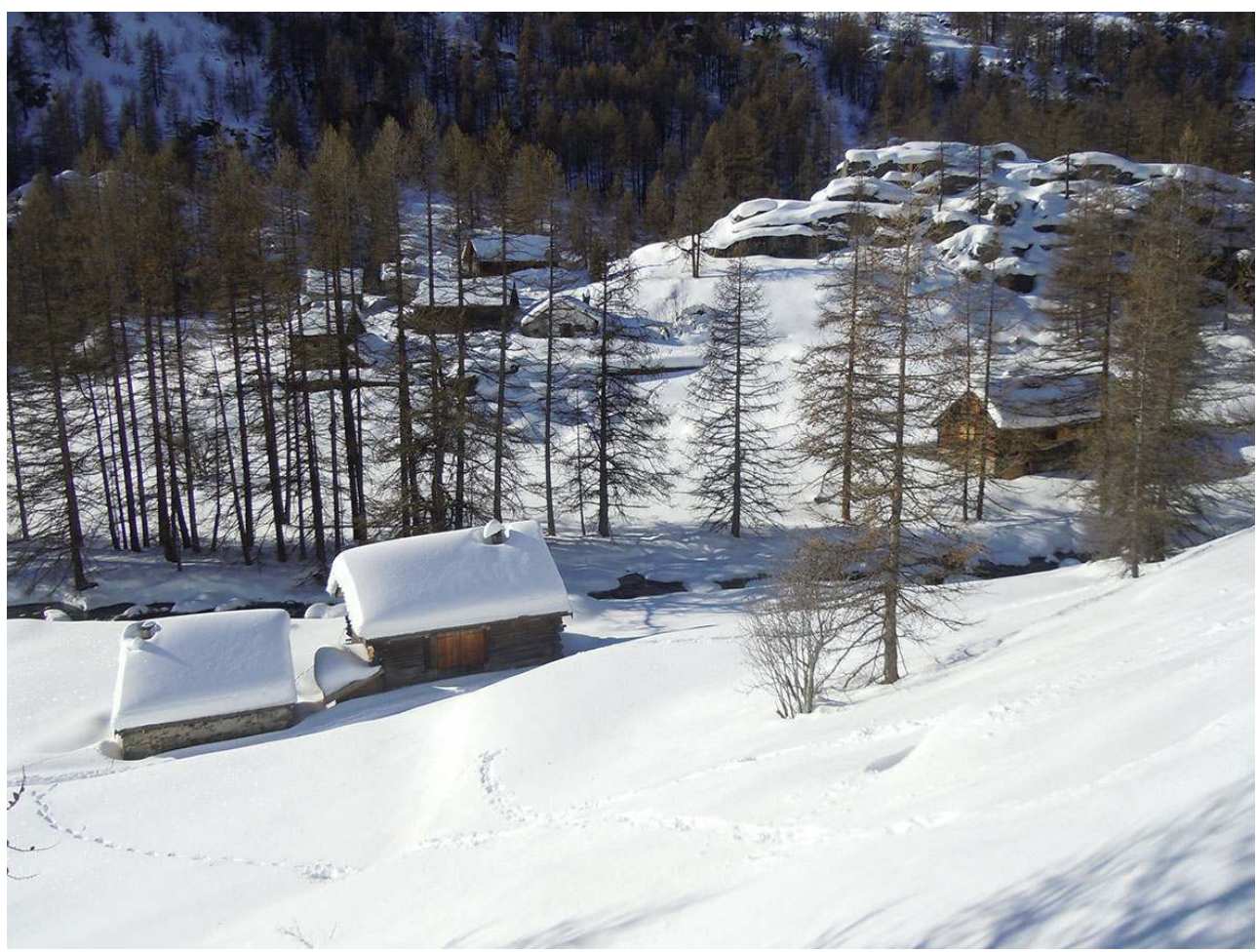

NÉVACHe, haUte VALLÉE de LA CLARÉE, SITE INSCRIT EN 1943, CLASSÉ EN 1992.

Phot. Philippe Grandvoinnet, 2013. (C) UDAP 05.

En contraste avec l'urbanisation désordonnée qui a gagné les vallées alpines après la Première Guerre mondiale, les hameaux d'altitude ont révélé, dès le milieu du xxe siècle, une apparente unité architecturale. Cette impression d'harmonie architecturale résulte de plusieurs facteurs: emploi exclusif de matériaux prélevés localement, techniques constructives simples, répétition de quelques modèles ancrés dans la tradition, lisibilité de la fonction de chaque terroir (champs, prés de fauche, alpages, forêts), opposition entre la montagne jardinée et la montagne sauvage qui en constitue l'écrin naturel ${ }^{16}$. Les géographes du début du xxe siècle, tel Raoul Blanchard (1877-1965), ont expliqué en quoi ces paysages résultaient d'un équilibre fragile obtenu de haute lutte par des générations de paysans. Leur transformation, accélérée par la révolution agricole et l'essor du ski, a été vue comme une menace ${ }^{17}$. Les clubs alpins et les acteurs du tourisme naissant (à commencer par les compagnies de chemin de fer, tel le Paris-Lyon-Méditerranée) s'en sont fait les porte-paroles en diffusant l'image d'une montagne préservée, source de bienêtre, d'évasion et d'authenticité. En protégeant in situ ces fragments de paysages agropastoraux, l'administration des Beaux-Arts, alors en charge de la protection des sites, s'inscrit dans la démarche scientifique de description, d'analyse et d'échantillonnage de la montagne engagée à la fin du XIX ${ }^{e}$ siècle, et dans laquelle les ethnographes s'illustrent en 
sauvegardant les éléments d'une culture agropastorale qui disparaît sous leurs yeux (mobilier et objets du quotidien notamment). La création en 1906 par Hippolyte Müller (1865-1933) du Musée dauphinois à Grenoble est exemplaire à ce titre. L'administration, qui s'intéresse dès cette date, mais surtout à partir de 1930, à la protection des paysages, accorde à ces espaces de haute montagne et aux éléments de patrimoine qui les composent (foncier, bâti, pratiques agricoles) une valeur nouvelle, fondée non plus seulement sur la valeur agronomique des terrains mais sur leurs qualités esthétiques, voire culturelles, en tant que témoignage de traditions montagnardes anciennes. Cet intérêt est d'autant plus vif que l'ethnographie, qui s'intéresse alors aux cultures populaires nationales, a mis en lumière la diversité des traditions d'une vallée à l'autre et la richesse des patrimoines à l'échelle du massif alpin.

11 Jean-Paul Guérin soulevait en 1996 la difficulté posée par l'évolution de la notion de patrimoine qui, «d'un sens matériel et privé ", tend "à un sens immatériel, public et métaphorique $»^{18}$. En montagne, où la propriété comme les usages peuvent être aussi bien individuels que collectifs, cette distinction prend un sens particulier. Lorsqu'un ensemble de biens privés (terrains, bâtiments, troupeaux) finissent par former un patrimoine collectif auquel la communauté dans son ensemble est attachée, la question de sa pérennité n'est posée qu'en termes d'usages. Traditionnellement, les conflits potentiels entre la jouissance privée d'un bien et son appartenance symbolique à un patrimoine collectif était résolue au sein de la communauté en se fondant sur les usages, ou en faisant appel à une autorité extérieure qui se prononçait en fonction de considérations légales mais aussi de l'intérêt collectif. Jusqu'au début $\mathrm{du} \mathrm{xx}^{\mathrm{e}}$ siècle, l'exploitation agropastorale de la montagne, fondée sur un usage intensif et des pratiques éprouvées (quoique variables d'une communauté à l'autre) assurait le lien entre la préservation du patrimoine privé et celle du patrimoine symbolique de la communauté. Les mutations opérées au $\mathrm{xx}^{\mathrm{e}}$ siècle ont fragilisé cet équilibre: la valeur patrimoniale que l'on accorde aujourd'hui aux paysages de haute montagne est indissociable des pratiques agropastorales traditionnelles qui les ont modelés et que l'on tente de prolonger, bien qu'elles aient considérablement évolué. Les moutons paissent toujours en été dans les alpages mais les champs ne sont plus guère fauchés, encore moins cultivés; on ne monte plus pour l'été dans le chalet d'alpage familial - si ce n'est pour les loisirs - et l'attention portée à la perpétuation des paysages, sans les pratiques qui en assurent l'entretien, ne mène nulle part. Pour cette raison, les logiques de simple protection ont progressivement évolué vers des logiques de mise en valeur, par exemple au travers des parcs ${ }^{19}$, dont les chartes ne se contentent pas d'identifier ce qui fait patrimoine mais définissent des stratégies d'action pour le maintenir vivant et en assurer le renouvellement. Plus récemment, la charte foncière agricole des Hautes-Alpes ${ }^{20}$ reconnaît que «les espaces entretenus par l'activité agricole sont de véritables atouts pour le patrimoine montagnard » et ambitionne de mieux intégrer les enjeux agricoles - notamment dans les secteurs sensibles de haute montagne - aux politiques d'aménagement du territoire en lien avec les agriculteurs et les acteurs fonciers (SAFER notamment).

\section{Chalets d'alpage et bâtiments d'estive}

Le patrimoine de l'agropastoralisme est ténu et parfois difficile à identifier. L'inventaire topographique a été réalisé dans les cantons du nord des Hautes-Alpes (Briançonnais, Queyras, haut Embrunais) à partir des années 1970 par le service de l'Inventaire général 
de Provence-Alpes-Côte-d'Azur. Ce travail a été mené jusque dans les plus hauts alpages, tels ceux de Cervières, de la vallée de la Clarée ou du Queyras, et diffusé auprès d'un large public via la base Mérimée et une publication récente ${ }^{21}$. Les résultats de cette étude viennent aujourd'hui enrichir les documents d'urbanisme ${ }^{22}$ mais n'ont suscité que de trop rares mesures de protection. Dans le Queyras, par exemple, où l'on trouve un type d'habitat propre à chaque vallée, seule une maison rurale est protégée au titre des monuments historiques (commune de Ceillac) ${ }^{23}$. À Saint-Véran, perché si haut dans la montagne que le village permanent lui-même est posé sur l'alpage, aucune " fuste $^{24}$ " n'est protégée, alors que ces constructions en fûts de mélèze assemblés constituent un type architectural unique, précisément codifié par une tradition qui remonte au XVII siècle au moins et parfaitement adapté aux pratiques agricoles locales (fig. 6). Une zone de protection du patrimoine architectural et urbain (ZPPAU, devenue "paysagère » en 1993 et transformée depuis en site patrimonial remarquable) a toutefois été créée en 1987 à Saint-Véran : elle couvre le village et le terroir agricole et naturel qui en forme l'écrin paysager et interdit la démolition ou la dénaturation des fustes anciennes ${ }^{25}$.

Figure 6

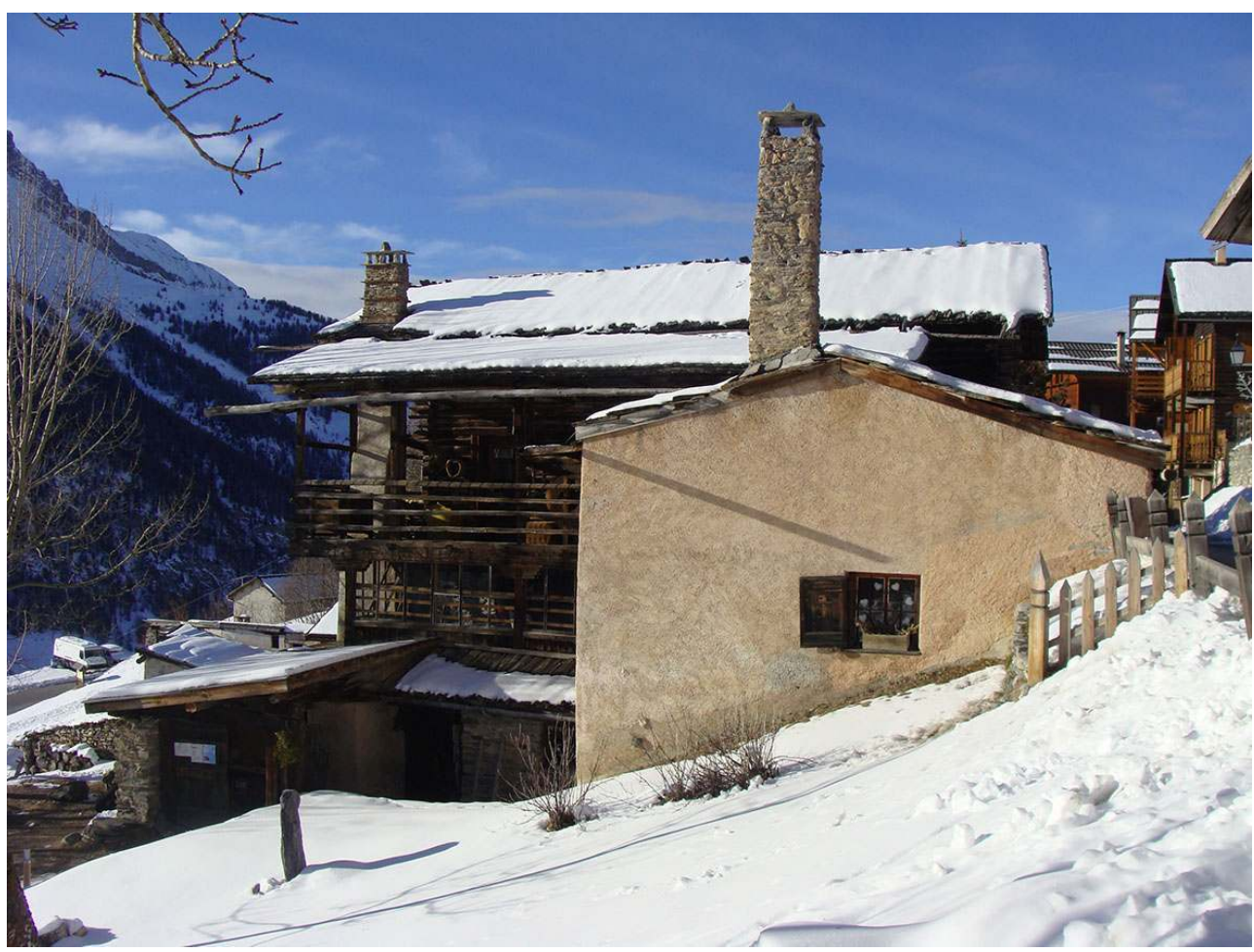

SAINT-VÉRAN, ANCIENNE FUSTE DITE “ MAISON DU SOUM », 1641.

Phot. Philippe Grandvoinnet, 2014. (C) UDAP 05.

13 Les hameaux saisonniers, chalets d'alpage et autres bâtiments d'estive constituent un patrimoine plus fragile encore car ils sont dispersés dans l'alpage et ont perdu leurs fonctions d'origine. Dans les Hautes-Alpes, où le système de la « petite montagne » était le plus courant, certains alpages comptent plusieurs dizaines de granges, chalets d'alpage ou cabanes pastorales, groupés en hameaux ou dispersés sur la totalité du plan ${ }^{26}$. Lorsque l'éloignement des alpages interdisait de rentrer quotidiennement au village, l'habitat saisonnier était installé à mi-pente et accueillait plusieurs membres de chaque famille une partie de l'été. Le village permanent de la vallée était alors dédoublé en altitude, sous 
une forme urbaine plus ou moins constituée (hameau d'estive) ou sous la forme de bâtiments isolés (fig. 7). La vocation de cet habitat saisonnier d'altitude était agricole (culture des céréales de printemps à la limite inférieure des alpages) autant que pastorale (protection des prés de fauche contre les troupeaux, irrigation, fenaison, transformation du lait, soin des bêtes ${ }^{27}$. Si les fouilles archéologiques entreprises dans le massif des Écrins ont mis au jour des vestiges de cabanes pastorales datant du Moyen Âge ${ }^{28}$, rares sont les bâtiments encore en place dont la construction ou la reconstruction est antérieure au XVIII ${ }^{\mathrm{e}}$ siècle $^{29}$. La haute montagne n'a pas toujours été telle que nous la connaissons aujourd'hui, presque vide d'habitants. La forte croissance démographique de la première moitié $\mathrm{du}$ xIX ${ }^{\mathrm{e}}$ siècle a donné lieu à une exploitation intensive de l'espace disponible et à la multiplication des habitats saisonniers à tous les étages de la montagne. Le patrimoine bâti dont nous héritons aujourd'hui résulte de ce double mouvement d'exploitation et de construction intenses, suivies d'un abandon progressif entre 1850 et 1970 sous les effets conjugués de l'exode rural, du sur-pâturage et de la dégradation des sols. Bien que pour partie ruinés, ces bâtiments n'en structurent pas moins fortement les alpages et, parmi les manifestations encore visibles des pratiques agropastorales, ils sont de celles auxquelles les habitants sont le plus attachés dans la mesure où elles s'inscrivent dans une histoire familiale.

Figure 7

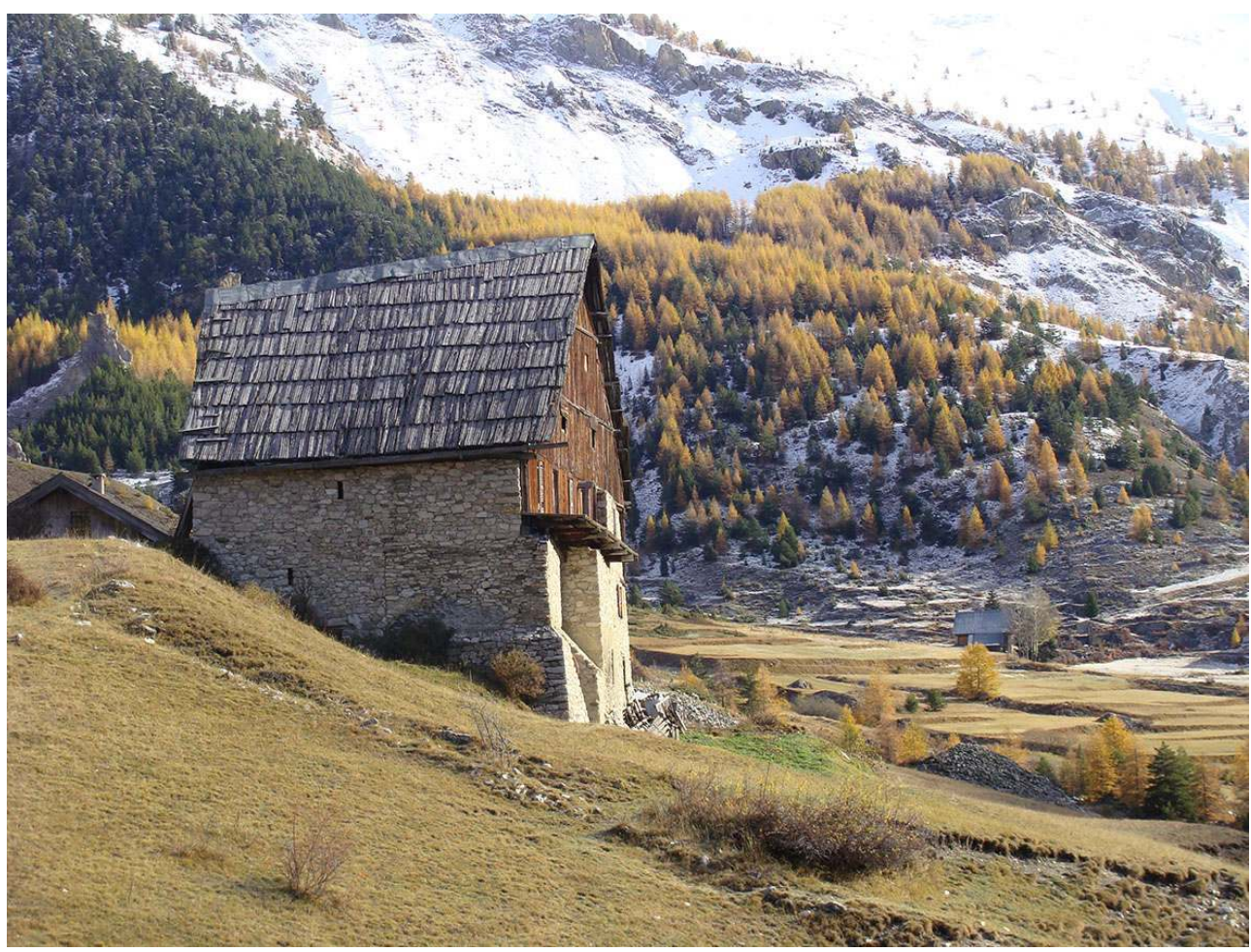

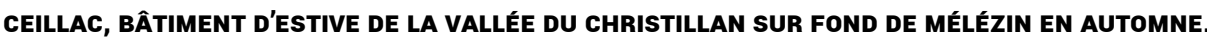

Phot. Philippe Grandvoinnet, 2014. (C) UDAP 05.

Dans sa version initiale de 1985 , la loi «Montagne ${ }^{30}$ " posait en principe absolu l'inconstructibilité des zones naturelles et agricoles, interdisant jusqu'à la restauration des chalets d'alpage et bâtiments d'estive isolés. Modifiée en 1994 pour intégrer cet enjeu patrimonial, la loi permet leur stricte restauration, après autorisation préfectorale, dans un objectif de « mise en valeur du patrimoine montagnard ${ }^{31} »$. Dans les Hautes-Alpes, les 
projets de restauration sont instruits par l'unité départementale de l'Architecture et du Patrimoine (UDAP) et examinés par la commission départementale de la Nature, du Paysage et des Sites (CDNPS). L'extension d'un bâtiment existant n'est admise que dans le cadre d'une activité agricole. Cinq cents dossiers ont été traités en vingt ans dans le département et les demandes soulèvent parfois des questions de doctrine : en effet, les bâtiments restaurés sont en majorité destinés aux loisirs (l'aménagement d'une bergerie ou d'une cabane d'alpage dans un bâtiment ancien reste l'exception) et doivent néanmoins conserver un aspect traditionnel (matériaux, finitions, dimension des fenêtres, traitement des espaces extérieurs, etc.). La loi Montagne a trouvé un grand nombre de hameaux d'estive dans un état de semi-abandon avec des bâtiments partiellement ou totalement ruinés. Dans le Briançonnais, le vallon des Combes est traditionnellement le lieu d'estive pour les villages de Puy-Saint-André et Puy-SaintPierre. Le hameau comptait déjà une quarantaine de maisons et granges en $1468^{32}$ et le cadastre de 1841 en répertorie plus de 80 , à son apogée démographique: il n'en reste aujourd'hui que 35. Beaucoup de chalets ont été restaurés; les dents creuses ont été transformées en jardins ou en terrasses d'agrément. Dans le Queyras, le hameau de PréRoubaud (commune d'Abriès) comptait une cinquantaine de bâtiments en 1825, plus que 6 en 2014. Afin de limiter la pression anthropique en alpage, la loi Montagne ne permet de restaurer que les bâtiments justifiant d'un intérêt patrimonial ; la volumétrie du bâtiment à restaurer doit donc être suffisamment lisible pour permettre la restitution fidèle des dispositions d'origine. Après plusieurs décennies d'abandon, les restaurations réalisées au cours des vingt dernières années ont redonné un aspect vivant aux alpages, toujours entretenus pas les troupeaux en estive (fig. 8).

Figure 8

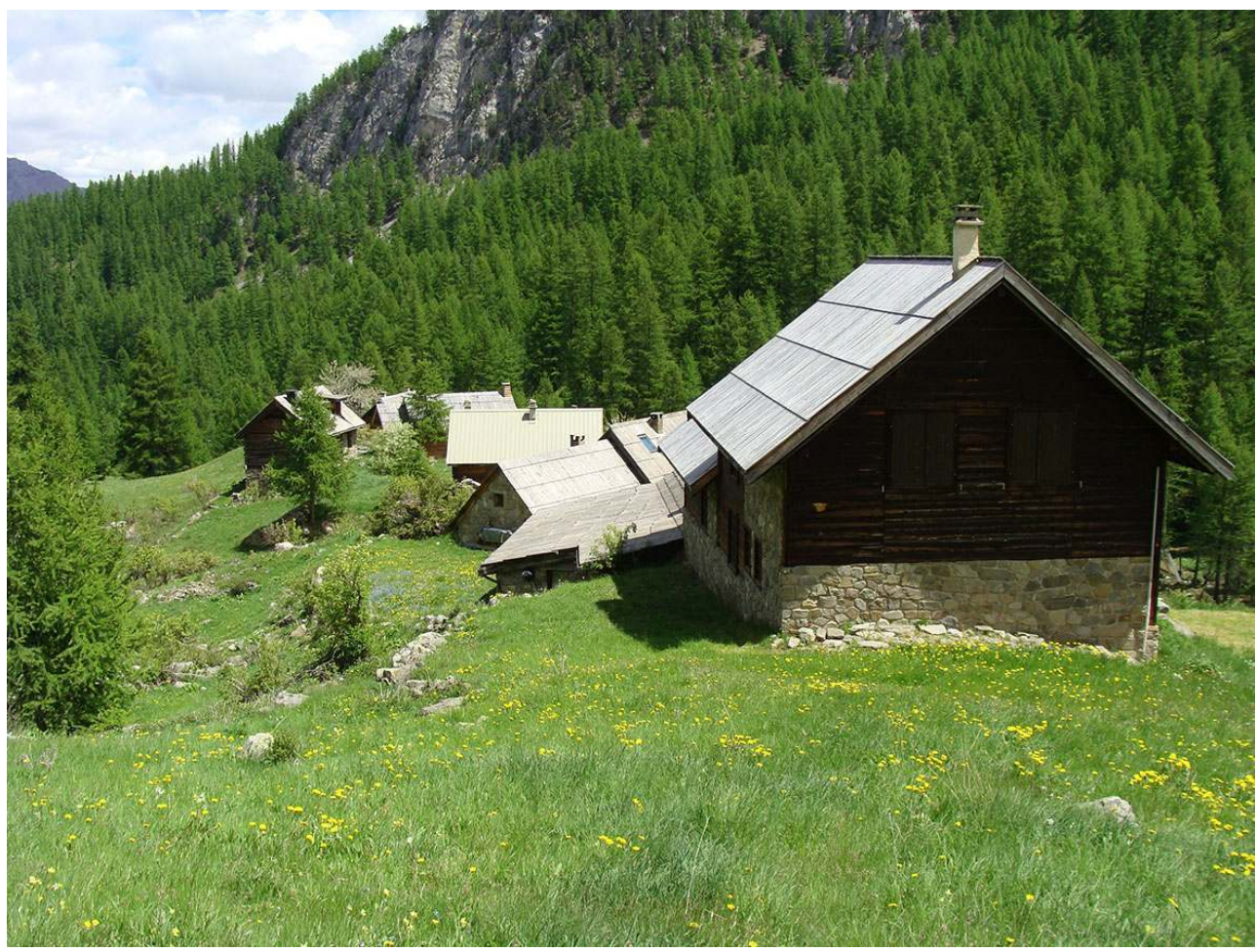

PUY-SAINT-VINCENT, ENSEMBLE DE CHALETS RESTAURÉS AU HAMEAU DE NAREYROU.

Phot. Philippe Grandvoinnet, 2015. (c) UDAP 05. 


\section{Les fermes de la Reconstruction : nouveaux patrimoines agropastoraux}

15 Le patrimoine vernaculaire n'est pas le seul à témoigner des pratiques agropastorales en montagne : la Reconstruction des années 1945-1955 a laissé sa marque jusque dans les hautes vallées. Une soixantaine de villages ont été sinistrés au cours de la bataille des Alpes, en juin 1940, puis du repli de l'armée allemande, en 1944-1945. Les combats ont été particulièrement violents sur la frontière avec l'Italie et plusieurs villages haut-alpins ont été entièrement détruits, parmi lesquels Cervières, Abriès et Ristolas (Briançonnais/ Queyras ${ }^{33}$. Lorsque la reconstruction fut planifiée, en 1945, la question de l'amélioration des techniques de culture et d'élevage et de modernisation de l'habitat fut posée. Placés sous la tutelle du ministère de la Reconstruction et de l'Urbanisme (MRU), les architectes en charge de la reconstruction des villages sinistrés développèrent en montagne les principes de l'urbanisme moderne formulés pendant l'entre-deux-guerres. Ils mirent à profit la situation de «table rase " pour restructurer les bourgs (voirie, orientation des bâtiments) et réorganiser les fermes selon de nouveaux principes agricoles et hygiéniques (séparation des hommes et des animaux, circulations intérieures, stockage du foin, etc.). Les populations étaient toutefois restées attachées à l'identité architecturale des villages anciens et les architectes se sont inspiré des matériaux et des modes de construction vernaculaires pour élaborer une architecture à la fois fonctionnelle et influencée par les formes traditionnelles ${ }^{34}$ (fig. 9). Les quartiers ou villages reconstruits constituent des ensembles homogènes qui, en raison de leurs références à l'architecture vernaculaire, s'insèrent harmonieusement dans leur contexte rural. Élaborées en s'appuyant sur une analyse fine des pratiques agricoles en vigueur à la fin de la guerre, ces réalisations ont contribué au renouvellement des patrimoines de haute montagne. 


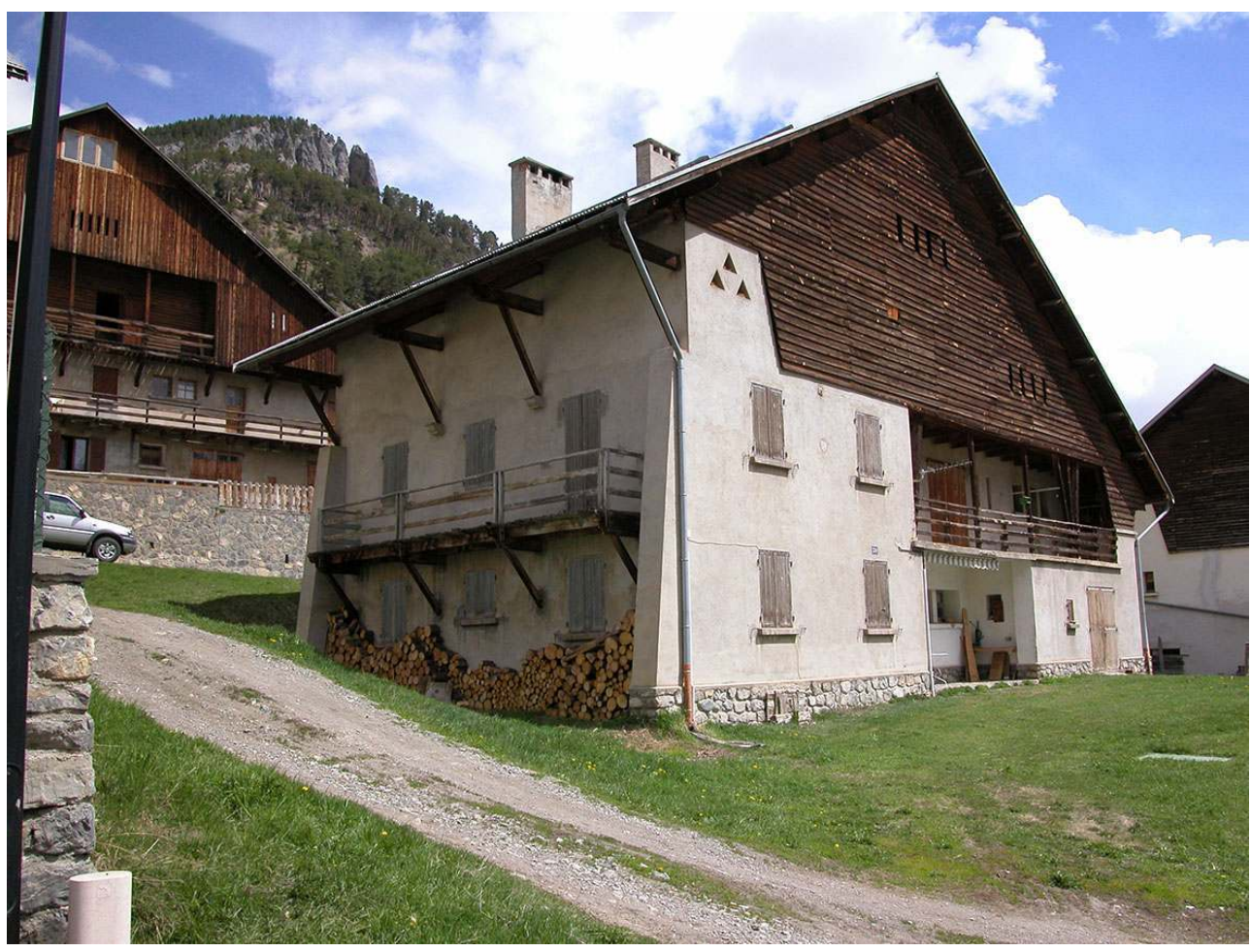

CERVIÈRES, FERME DE LA RECONSTRUCTION LABELLISÉE « ARCHITECTURE CONTEMPORAINE REMARQUABLE ".

PHOT. HÉLÈNE RIBLET, 2007. @ UDAP 05.

Dans le cadre de la campagne d'attribution du label «Patrimoine du $\mathrm{xx}^{\mathrm{e}}$ siècle », devenu «Architecture contemporaine remarquable», aux œuvres architecturales les plus significatives du siècle écoulé, la direction régionale des Affaires culturelles de ProvenceAlpes-Côte d'Azur a reconnu les qualités urbaines et architecturales de plusieurs villages reconstruits dans les Hautes-Alpes comme Cervières (Briançonnais, $1500 \mathrm{~m}$ d'altitude) ${ }^{35}$ et Ristolas (Queyras, $1600 \mathrm{~m}$ d'altitude) ${ }^{36}$. La mise en valeur du patrimoine de l'agropastoralisme peut contribuer à fonder un projet de territoire. À Cervières, la diversité de ces patrimoines façonne l'identité de la commune : le bourg ancien, bien que partiellement détruit pendant la guerre, conserve de beaux exemples d'architecture vernaculaire dont une maison inscrite au titre des monuments historiques ${ }^{37}$. Les fermes de la Reconstruction, labellisées "architecture contemporaine remarquable", constituent un ensemble architectural parfaitement intégré au site. La vallée des Fonts, frontalière de l'Italie, offre un paysage de haute montagne remarquable dont l'une des principales qualités est la présence de nombreux chalets d'alpage (fig. 10). Consciente de la valeur de son patrimoine, la municipalité réfléchit à la création d'un site patrimonial remarquable (SPR). Fondé sur l'analyse des pratiques et des patrimoines de l'agropastoralisme, ce document doit constituer le cadre d'une action concertée (paysage, urbanisme, architecture) qui permettra de maintenir vivants les patrimoines de la communauté. 


\section{Figure 10}

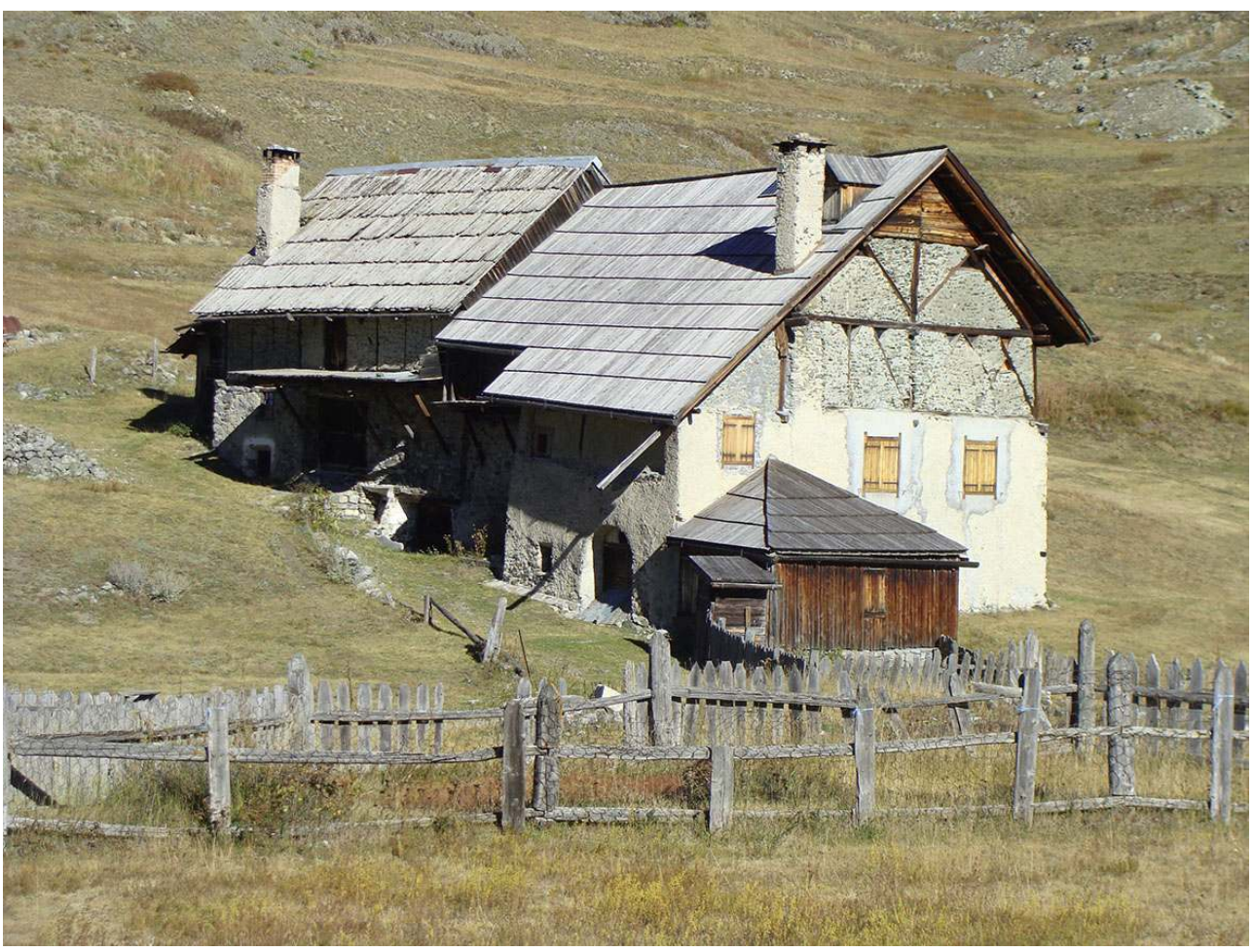

Cervières, chalet d'alpage au hameau de lachau, vallée des fonts.

Phot. Philippe Grandvoinnet, 2014. (C) UDAP 05.

\section{NOTES}

1. - Philippe Grandvoinnet a été chef de l'unité départementale de l'Architecture et du Patrimoine des Hautes-Alpes de 2012 à 2015.

2. - CARRIER, Nicolas, MOUTHON, Fabrice. Paysans des Alpes. Les communautés montagnardes au Moyen Âge. Rennes : PUR, 2010.

3. - JOURDAIN-ANNEQUIN, Colette, DUCLOS, Jean-Claude (dir.). Aux origines de la transhumance. Les Alpes et la vie pastorale d'hier à aujourd'hui. Paris : Picard, 2006.

4. - THÉNOZ, Michel. «Les paysages humanisés du parc national des Écrins ». Revue de géographie de Lyon, 1982, nº 4, p. 393.

5. - Voir MALLÉ, Marie-Pascale (dir.). L'Habitat du nord des Hautes-Alpes. Patrimoine architectural et mobilier. Aix-en-Provence/Gap : Association pour le patrimoine de Provence/Société d'études des Hautes-Alpes, coll « Cahiers du patrimoine, 55 », 1999, p. 83-103.

6. - JOURDAIN-ANNEQUIN, Colette, DUCLOS, Jean-Claude (dir.). Aux origines de la transhumance. Op. cit., p. 69.

7. - CARRIER, Nicolas, MOUTHON, Fabrice. Op. cit., p. 242-245.

8. - MALLÉ, Marie-Pascale. Op. cit., p. 87. 
9. - Ce témoignage a été publié dans le Guide de restauration des bâtiments d'estive dans les HautesAlpes. STAP des Hautes-Alpes, Gap, 2005. Les mots suivis d'un * correspondent à des expressions propres à l'estive : se changer: déménager pour l'estive; la montagne: la maison d'estive ou l'alpage ; tramois : trois mois ; plan : niveau de plancher dans un comble; vacherie : regroupement des troupeaux; village, pays : hameau d'altitude; trousse: grand filet garni de foin; ménagère : femme chargée du soin des animaux, de la traite, de la préparation du fromage et de la vie de la maison. En été, la ménagère effectuait chaque jour le trajet entre le village permanent et l'alpage pour effectuer un double travail : celui lié à l'estive, et celui qui demeurait en bas (repas de ceux qui n'étaient pas montés en début et fin d'estive, jardin du bas, etc.).

10. - MALLÉ, Marie-Pascale. Op. cit., p. 83.

11. - CARRIER, Nicolas, MOUTHON, Fabrice. Op. cit., p. 251.

12. - Le pin cembro (Pinus cembra), présent dans tout l'arc alpin, est l'essence emblématique de la haute montagne, car il trouve des conditions optimales à une altitude comprise entre 1700 et 2500 mètres. Sa croissance est lente mais il donne un bois de qualité apprécié en menuiserie et en sculpture.

13. - Loi du 2 mai 1930 ayant pour objet de réorganiser la protection des monuments naturels et des sites à caractère artistique, historique, scientifique, légendaire ou pittoresque. Cette loi est toujours en vigueur; elle succède à la loi du 21 avril 1906 organisant la protection de sites et monuments naturels de caractère artistique.

14. - Rapport général de protection au titre des sites, 1948, cité par les fiches DREAL de protection des sites, 2006.

15. - La politique de restauration des terrains en montagne (RTM) est instaurée en France dans les années 1860.

16. - THÉNOZ, Michel. Art. cit., p. 393-394.

17. - Ibid., p. 395.

18. - GUÉRIN, Jean-Paul. «Patrimoine et architecture vernaculaire». Dans CLIVAZ, Michel, BRUSSON, Jean-Paul (dir.). Patrimoine rural, architecture et paysage de l'arc alpin. Actes du colloque de Sion des 20, 21 et 22 juin 1996, Genève/Sion : Institut d'architecture de l'université de Genève/ Institut universitaire Kurt-Bösch, 1998, p. 63.

19. - Création du parc national des Écrins en 1973 et du parc naturel régional du Queyras en 1979. La Charte du parc national des Écrins (2013) compte plusieurs orientations touchant à l'agropastoralisme : "faire vivre une culture commune », " préserver et valoriser le patrimoine bâti rural », " soutenir la gestion globale des alpages ». En cœur de parc, l'un des objectifs de la charte est de " renforcer la gestion des ressources agropastorales et forestières ".

20. - Voir sur le site : charte foncière agricole des hautes-Alpes [consulté le 19/10/2017]. Signée le 15 février 2015 entre la chambre d'agriculture, la préfecture, le conseil général, l'association des Maires de France, l'association des Maires ruraux et la SAFER.

21. - MALLÉ, Marie-Pascale (dir.). L'Habitat du nord des Hautes-Alpes. Op. cit.

22. - L'article L. 123-1-5-III- ${ }^{\circ}$ du code de l'urbanisme permet "d'identifier et de localiser les éléments de paysage et délimiter les quartiers, îlots, immeubles, espaces publics, monuments sites et secteurs à protéger, à mettre en valeur ou à requalifier pour des motifs d'ordre culturel, historique, architectural ou écologique ». Cet outil offre un champ d'application extrêmement large puisqu'il peut concerner des édifices isolés et des ensembles bâtis, mais aussi des éléments naturels ou paysagers (points de vue, arbres remarquables, cheminements, jardins, etc.).

23. - Voir dans la base Mérimée : notice IA05000552.

24. - Structure de fûts de mélèze entrecroisés et assemblés à quart de bois aux extrémités, correspondant au volume de la grange (MALLÉ, Marie-Pascale. L'Habitat du nord des Hautes-Alpes. Op. cit., p. 416).

25. - PERRON, Claude. Saint-Véran. Zone de protection du patrimoine architectural et urbain. Aix-enProvence : Édisud, 1990. 
26. - Les inventaires réalisés par certaines communes permettent de donner une estimation assez précise des bâtiments encore debout : 60 à Ceillac, 130 à Arvieux, 150 à Cervières, plus de 300 à Névache.

27. - MALLÉ, Marie-Pascale. Op. cit., p. 84.

28. - CARRIER, Nicolas, MOUTHON, Fabrice. Op. cit., p. 245.

29. - Toutefois, la rareté du bois dans certaines vallées a conduit à un recyclage méticuleux des matériaux lors des reconstructions successives, de sorte que les bois qui composent ces bâtiments sont souvent plus anciens que les bâtiments eux-mêmes, parfois médiévaux. ÉDOUARD, Jean-Louis. "Longue chronologie de cernes du mélèze et occupation humaine depuis plus de mille ans dans la vallée de la Clarée (Briançonnais, Alpes françaises)». Dans TZORTZIS, Stéfan, DELESTRE, Xavier (éd.). Archéologie de la montagne européenne. Actes de la table ronde internationale de Gap, Musée-muséum départemental de Gap (Hautes-Alpes), 29 sept.- er $^{\text {er }}$ oct. 2008. Paris/Aix-en-Provence: Errance/Centre Camille-Jullian, coll. «Bibliothèque d'archéologie méditerranéenne et africaine, 4 », 2010, p. 325-333.

30. - Loi $n^{\circ} 85-30$ de janvier 1985 relative au développement et à la protection de la montagne, dite loi Montagne.

31. - Sur les aspects juridiques et de procédure, voir JOYE, Jean-François. « Le chalet d'alpage : nouvel enjeu d'urbanisation des pâturages ». Construction, urbanisme, mars 2015, p. 8-14.

32. - CARRIER, Nicolas, MOUTHON, Fabrice. Op. cit., p. 243.

33. - LYON-CAEN, Jean-François, LYON-CAEN, Jean-Jacques, BAZIN, Luc, SCHNEEGANS, Guy. La Reconstruction dans les Alpes françaises, 1945-1955, architecture et urbanisme. Rapport de recherche non publié, ministère de l'Équipement, direction de l'Architecture, École d'architecture de Grenoble, 1983.

34. - Parc naturel régional du Queyras. Ristolas (Hautes-Alpes), un village reconstruit au $\mathrm{XX}^{e}$ siècle (1945-1965). Étude documentaire et inventaire de la production architecturale et urbaine du village reconstruit de Ristolas en vue de sa labellisation «Patrimoine $\mathrm{du} \mathrm{xx}^{\mathrm{e}}$ siècle ", rapport de synthèse, décembre 2014.

35. - Voir la fiche label architecture contemporaine remarquable.

36. - Voir la fiche label architecture contemporaine remarquable.

37. - Voir dans la base Mérimée : notice PA05000018.

\section{RÉSUMÉS}

Les traditions agropastorales du cœur des Alpes ont fortement évolué au cours du $\mathrm{xx}^{\mathrm{e}}$ siècle, mais des fragments en restent vivaces. Face à un patrimoine culturel dont les fondements immatériels ne sont plus immédiatement perceptibles, les paysages (en tant qu'objets culturels), le tissu agricole et les métiers dont il est le support, les formes urbaines (résultat d'une accumulation séculaire) et l'architecture vernaculaire (vecteur d'identité) restent les marqueurs d'un mode d'habiter la montagne. Dans les Hautes-Alpes, les pratiques d'estive se perpétuent, mais les patrimoines qui lui sont associés ont connu une période d'abandon puis de reconquête. Les paysages de montagne ont été façonnés par les pratiques agricoles dont ils constituent un témoignage vivant. Les hameaux d'alpage et les bâtiments d'estive isolés sont des constructions fragiles qui nécessitent un entretien constant; leur restauration assure la pérennité de ce patrimoine montagnard même s'ils ne sont désormais que rarement utilisés pour l'estive. Les 
patrimoines de l'agropastoralisme ont évolué avec les pratiques, comme en témoignent les fermes de la Reconstruction : bâties en haute montagne, elles offrent une typologie nouvelle fondée sur la modernisation des techniques au sortir de la guerre.

Agropastoral traditions have changed considerably in the Alps since the beginning of the twentieth century, but some of these traditions are still alive and prospering. Bearing witness to a cultural heritage of which the intangible roots are not easily perceptible, landscapes, understood as cultural objects, the agricultural fabric and the different crafts that maintain it, the built forms of dwellings resulting from the accumulations of centuries, vernacular architecture as a vector of identity, all these remain as indications of how the mountains were inhabited. In the Hautes-Alpes department, the practice of mountain pasturing during the summer ('estive') is still perpetuated, although the heritage associated with this practice has witnessed a period of abandonment and then reconquest. The mountains landscapes have been fashioned and sculpted by agropastoral practices and remain as a living witness to these practices. The buildings for the alpine pasturing and the isolated shelters for shepherds are fragile constructions requiring constant maintenance. Their restoration guarantees the survival of this mountain heritage, even if the buildings are only rarely used today for pasturing. The evolution of the heritage of agropastoralism is well illustrated by the farms of the reconstruction period after the Second World War. They offer a new typology based on post-war modernisation.

INDEX

Mots-clés : agropastoralisme, paysage, chalets, estive, alpage, montagne, Hautes-Alpes, Dauphiné, Briançonnais, Queyras, Embrunais, Ceillac, Cervières, Saint-Véran, Arvieux

Keywords : agropastoralism, landscapes, chalets, mountain pastures, alpage, mountain, HautesAlpes, Dauphiné, Briançonnais, Queyras, Embrunais, Ceillac, Cervières, Saint-Véran, Arvieux

\section{AUTEUR}

\section{PHILIPPE GRANDVOINNET}

Architecte et urbaniste de l'État, adjoint au sous-directeur de l'Enseignement supérieur et de la Recherche en Architecture, ministère de la Culture, chercheur associé EA 3400 ARCHE (Université de Strasbourg) philippe.grandvoinnet@culture.gouv.fr 